\title{
FE modeling and analysis of L4-L5 lumbar segment under physiological loadings
}

\author{
Bin Yu*, Chen Zhang, Chunyu Qin and Huaijiang Yuan \\ School of Computer Science and Technology Xidian University, Xi'an, Shaanxi, China
}

\begin{abstract}
.
BACKGROUND: Because of the complexity and construction, the failure rate of lumbar surgery is still high. It is necessary to find a solution to help improve the accuracy and safety of surgery.

OBJECTIVE: Construction and stress analysis of lumbar spine.

METHODS: The generated FE model based on CT scan images was validated and used to investigate the motion, and stress/strain of the vertebrae under different physiological loadings. A degenerated model was also simulated by changing the materials properties (E and Poisson's Ratio) of the intervertebral disc nucleus and annulus fibers from $1.0 \mathrm{~N} / \mathrm{mm}^{2}$ to $3.0 \mathrm{~N} / \mathrm{mm}^{2}$, and 0.42 to 0.45 , respectively.

RESULTS: When annulus fibrosis is degenerating or under ictal external loadings, the annulus fibrosis will be in a state of protrusion and there will potential for other pathological changes, such as herniation of the nucleus pulpous.

CONCLUSION: The effects of these changes in the corresponding motion and stress/strain of the spinal motion segments were investigated with some conclusions drawn in relation to the normal model for future application of medical diagnosis and surgical treatment.
\end{abstract}

Keywords: Lumbar spine, motion, stress/strain, physiological loadings

\section{Introduction}

FEA method is an effective method used to calculate approximate solution of complex differential equation, and it is fundamental to digital science and technology theory. This paper replaces a complicated function with a combination of massive simple function. This allows for any complex geometry structure to be easily analyzed and calculated by employing the FEA method. The ease at which FEA method results are interpreted is the reason why, this method is usually used to solve more complex practical problems that are concerned with material properties and shape [1].

The complex nature of the human spinal structure and components often create problems for gathering information when traditional methods are used. The finite element analysis method can be utilized with a variety of geometric units in order to populate and segment the model. Consequently, the FEA method is the most convenient method for finding global solution of the problem. This paper begins by describing the geometry model construction of humans lumbar vertebrae by utilizing matching cubes and volume rendering. The complete model of human spinal L4-L5 segments based on region growing algorithm and dividing the finite element into mesh is then described. Finally, the qualitative contours plots' simulation of displacement, stress and strain of the normal and degenerated model. These models are under the conditions of different stressed states and the validity verification of model data by contrast analysis are preceded after the addition of the soft tissue ligament for the model.

\footnotetext{
${ }^{*}$ Corresponding author: Bin Yu, School of Computer Science and Technology Xidian University, Xi'an, Shaanxi, China. E-mail: yubin@mail.xidian.edu.cn.
} 


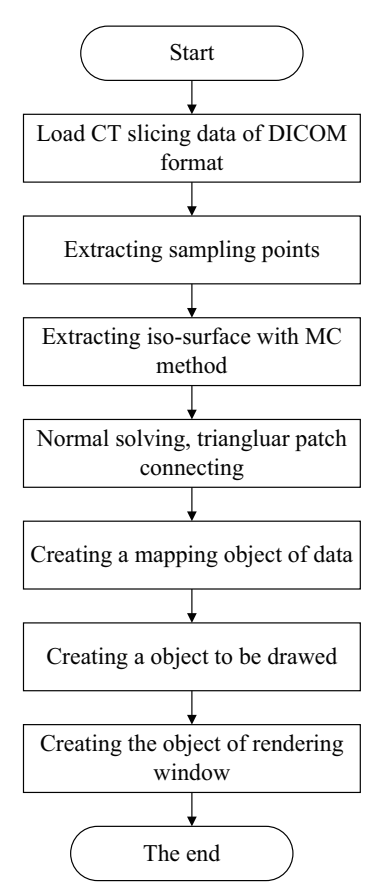

Fig. 1. Process of VTK MC surface rendering.

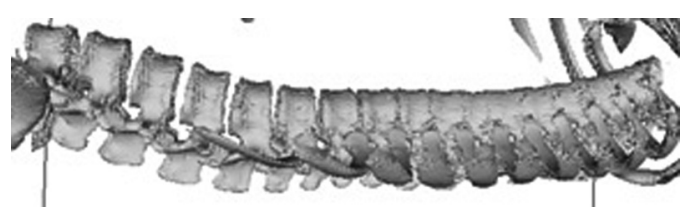

Fig. 2. The facial model of spine.

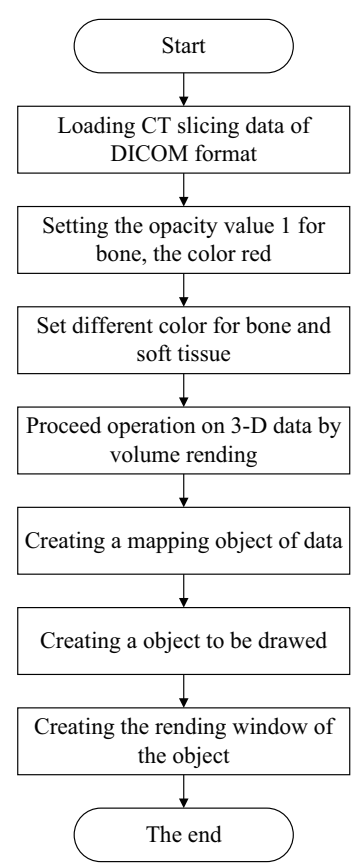

Fig. 3. The process of volume rendering.

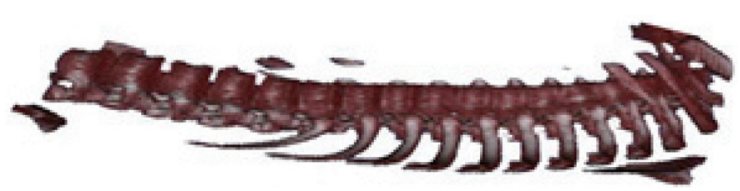

Fig. 4. The volume model of spine.

\section{Construction of human lumbar vertebrae 3-D model}

Through the use of CT image data, the lumbar vertebrae model is accomplished by adopting methods of matching cubes and volume rendering in this article. The VTK software developer kit is used to create a 3-D model. This kit uses the assembly line mode, in order to achieve matching cubes surface rendering [2], as shown in Fig. 1.

The results of generated surface model are shown in Fig. 2.

The VTK volume rendering process is shown in Fig. 3.

The generated volume model of the spine is as shown in Fig. 4.

The MC algorithm embodies the idea of partition, the algorithm's Iso-surface Extraction is extracted in every single voxel. The voxel of interesting this study is a cubic structure made by four corresponding pixels that are extracted from two consecutive CT images. For each single voxel, a triangular patch is used to close in the final required internal Iso-surface. The acquired Iso-surfaces are used to distinguish the boundary of the object. In the end, the 3-D model is constructed by contiguous Iso-surfaces.

The algorithm of volume rendering used in this paper is Ray-Projection. The procedure is shown as followed:

(1) Determine the 3-D data set based on the collected CT image sequence. 
(2) Classify data that are needed to be analyzed, according to the difference between data values. To better visualize the different tissues, color value and opacity of the data should be set before.

(3) Proceed equidistant sampling of data value, the ray starts off every pixel of the imaging plane, according to the direction of the setting in advance. The equidistant sampling will be proceeded on this ray in order, to acquire the color and opacity values of the sampling point. The linear interpolation should be proceeded three times based on the color value and opacity value of the eight voxels that are the closest from the sampling point.

(4) Generate the image. This process begins with the acquisition of the color value and opacity value of each equidistant sampling point of the Ray. Then the value accumulation should be conducted until the Ray is completely absorbed. When this process is done, the final image will be displayed on the screen.

Through the use of comparative techniques, it can be discovered that surface rendering clearly displays the external surface of the model, that the acquired images of the model are clear and smooth, and the shape is relatively life-like. Volume rendering allows the internal structure of the model to be maintained, and more clearly emphasize the internal characteristics. Both methods achieve the complete outline structure of the spine, but usually include some redundant information, such as rib fragment. Therefore, if a 3-D model of single vertebral section were wanted, the spine model must be incised with the bounding box algorithm [3]. However, because of the complex and irregular structure of the spine, relatively precise models cannot be achieved by adopting regular bounding box algorithm. When restricted to CT images, soft tissue, such as discs, cannot be reconstructed, with MC or volume rendering. In consequence, CT images are not well suited for finite element analysis. Here we explore the construction of a complete 3-D model that is suitable for FEA, based on the method of graphical segmentation.

\section{Construction of the finite element model of lumbar vertebrae}

The finite element model of lumbar vertebrae L4-L5 segments is generated by using simpleware and adopting region-growing algorithm which is incredibly linked.

The region-growing algorithm with confident link [4] must have the seed point artificially selected. The seed point is usually the geometric center of the target area. By setting a multiplication factor which can control the range of brightness, the cope of the grey value can be determined when these results are multiplied by the standard deviation of the pixels in a small area that contain a seed point. Further, the pixel within the scope will be joined into this area. To define a new range, repeated calculation of those pixels contained in the expanded area is required until there are no new pixels, or the number of promised iterations is over. In this text, the 3-D model is rebuilt with the confident region of different sectional drawing obtained following the segmentation of CT images by software simple ware and adding mask [5]. As a result of the limitation of hardware conditions, we make the lumbar vertebrae 3-D model of the L4-L5 segments grid-enabled and process them through mechanical analysis.

Here some operations need to be used on the model of L4-L5 segments, such as smoothing effect, meshing effect and assigning material properties. Usually material Poisson-ratio, elastic modulus and junction surface's properties should be set. The generated 3-D FE model of the L4-L5 segments is shown in Fig. 5. 


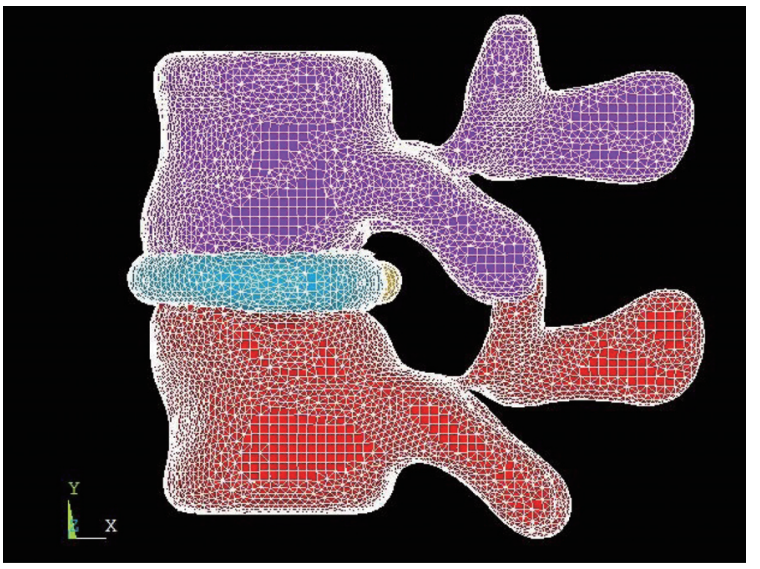

Fig. 5. The solid FE model of lumbar spinal L4-L5 segments.

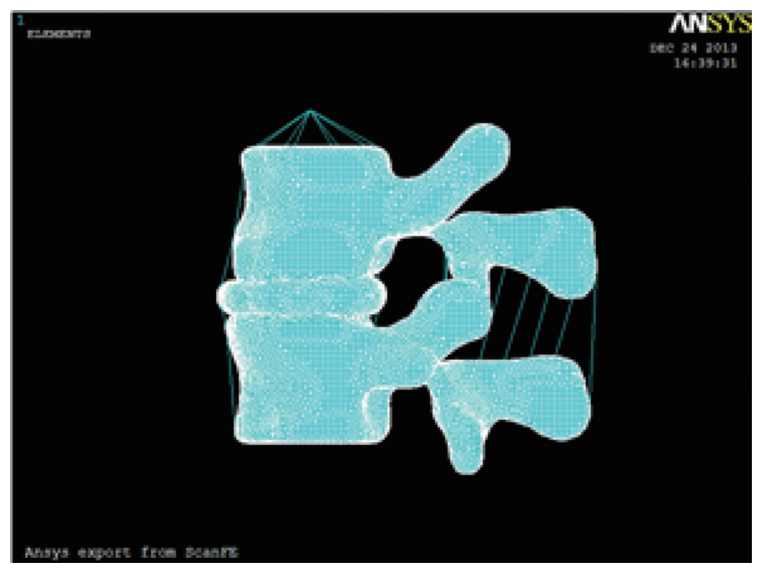

Fig. 6. The FE model of L4-L5 sections after ligament added.

\section{The mechanical analysis of the 3-D FE model of lumbar vertebrae L4-L5 sections}

\subsection{The choices of the ANSYS loading and the unit}

The basic steps of finite element analysis by utilizing ANSYS include the following: (1) the construction of the model; (2) the addition of the loading; (3) the post-processing of results. Before the model can be analyzed and calculated, some aspects of the model unit and the addition of loading need to be completed.

In order to simulate the solution of displacement and stress in the structure caused by external loading, statical analysis was conducted on the model of lumbar vertebrae. Each part of the defined vertebrae is a linear elastic, isotropic material, and the vertebral pulp is a solid, incompressible material. ANSYS provides various units that can process description of the model for the simulation accuracy of the hexahedral element, which is higher than that of tetrahedron element. A solid unit was chosen, such as SOLID45, to describe the model of lumbar vertebrae. The bar element can be simulated as a rope that is only capable of an extension function. This element is employed to simulate the ligament. Unit MPC184 is appropriate for adding stressor constraints, so this unit is chosen to exert various forces and moments, which are coming from different directions on models.

In the process of analysis and calculation in this paper, the exertion of corresponding constraints of degrees of freedom, that can be added on models and acted as boundary conditions, is required.

\subsection{The simulative construction of ligament}

The material properties of different ligaments were pulled from the primary literature [6-8] and are shown in Table 1.

The anterior longitudinal ligament(four units), posterior longitudinal ligament(four units), ligamentous Flava(two units), supraspinal ligament(one unit), interspinous ligament(four units) and capsular ligament(six units), are all simulated by adopting the unit Link 10. All units hold the feature of tension, but not that of pressure. In the first simulation, only the capsular ligament is added. The small joint is an integral part of the vertebral bodies. They achieve this effect through direct contact with the upper and lower articular in resistance to the rotation of movement. Additionally, they function well in maintaining 
Table 1

The material parameters of ligament

\begin{tabular}{lcccc}
\hline Material name & MOE/MPa & Poisson ratio & Sectional surface & Units \\
\hline Anterior longitudinal ligament & 20 & 0.3 & 75.9 & 4 \\
Posterior longitudinal ligament & 20 & 0.3 & 75.9 & 4 \\
Ligamentous flava & 19.5 & 0.3 & 8.7 & 2 \\
Supraspinal ligament & 11.6 & 0.3 & 75.7 & 1 \\
Interspinous ligament & 15 & 0.3 & 6.1 & 4 \\
Capsular ligament & 32.9 & 0.3 & 102.5 & 6 \\
\hline
\end{tabular}

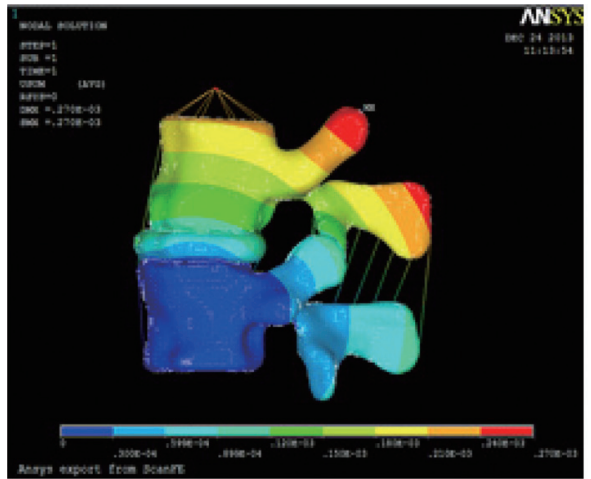

(a)

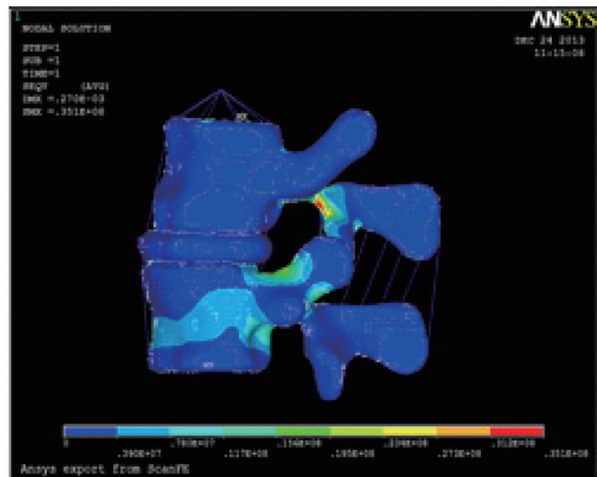

(b)

Fig. 7. (a) The qualitative displacement contours plot (b) The qualitative displacement contours plot. The simulated results of flexion movement.

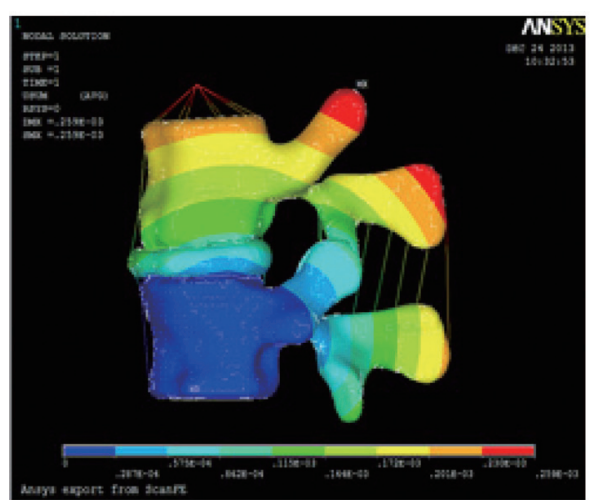

(a)

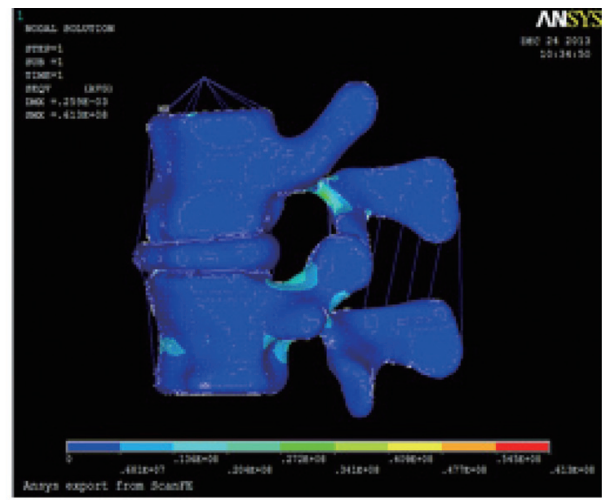

(b)

Fig. 8. (a) The qualitative displacement contours plot (b) The qualitative displacement contours plot. The simulated results of extension movement.

the distance between the surfaces of the two small joints. Therefore, to ensure the small joints correctly maintain normal movement of the vertebral bodies, the capsular ligament is needed, and a small joint should be manipulated as the contact model.

The generated FE model after the addition of the ligament is as shown in Fig. 6. 


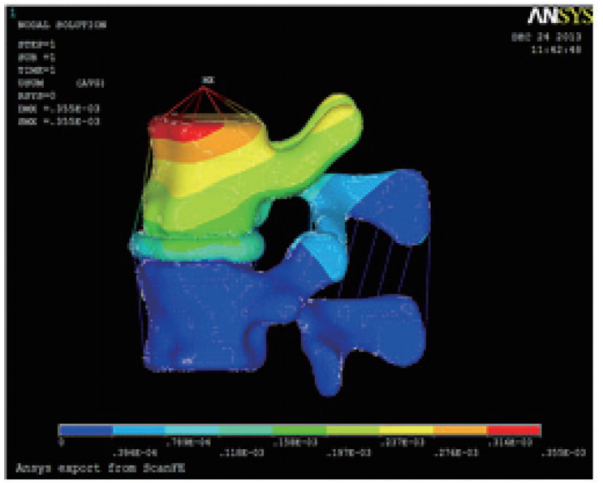

(a)

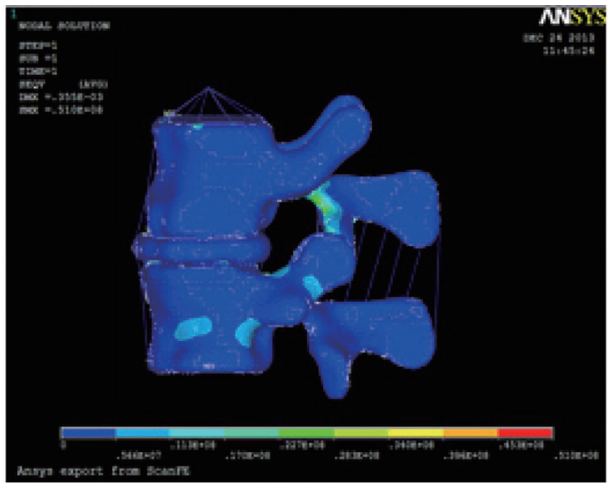

(b)

Fig. 9. (a) The qualitative displacement contours plot (b) The qualitative displacement contours plot. The simulated results of left flexion movement.

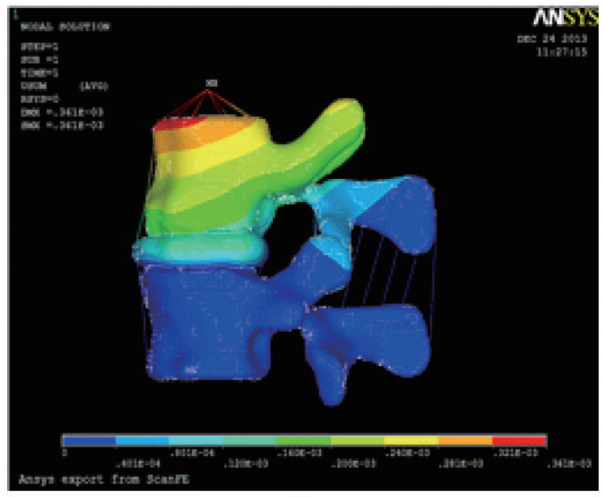

(a)

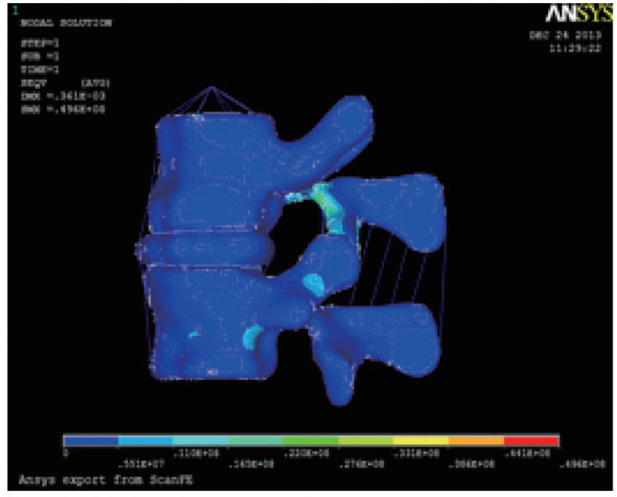

(b)

Fig. 10. (a) The qualitative displacement contours plot (b) The qualitative displacement contours plot. The simulated results of right flexion movement.

\section{Results and discussion}

\subsection{Stress analysis of L4-L5 sections in the normal model}

To get the displacement deformations and stress conditions of the model under different stress states, we secured the lower endplates of the L5 section of the vertebral body, and exerted surface traction of $200 \mathrm{~N}$ on the upper endplates of the L4 section of the vertebral body. This was done to simulate the natural load on the L4-L5 lumber sections caused by the human body. Further, five different stresses were simulated by loading the following boundary conditions. (1) To simulate flexion movement of the lumbar vertebrae, $200 \mathrm{~N}$ of force were exerted along the positive direction of the $x$-axis. (2) To simulate extension movement of the lumbar vertebrae, $200 \mathrm{~N}$ of force were exerted along the negative direction of the $x$-axis. (3) In order to simulate the left flexion movement of the lumbar vertebrae, $200 \mathrm{~N}$ of force were exerted along the positive $z$-axis. (4) To simulate the right flexion movement of the lumbar vertebrae, $200 \mathrm{~N}$ of force was exerted along the negative direction of $z$-axis. (5) To simulate the axial rotational movement of lumbar vertebrae, $20 \mathrm{~N} \cdot \mathrm{m}$ of torque was exerted along the $y$-axis. The achieved qualitative contours plots of displacement and stress are shown as followed figures (Figs 7-11). 


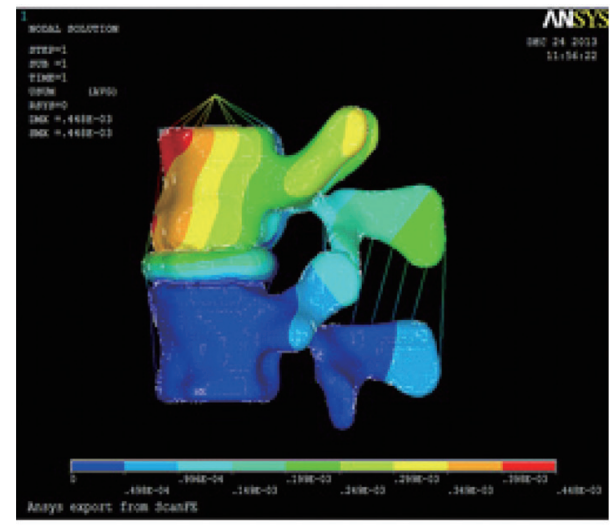

(a)

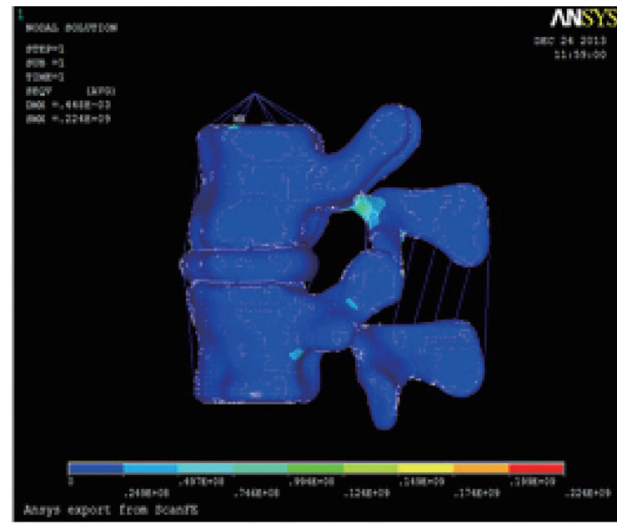

(b)

Fig. 11. (a) The qualitative displacement contours plot (b) The qualitative displacement contours plot. The simulated results of axial rotational movement.

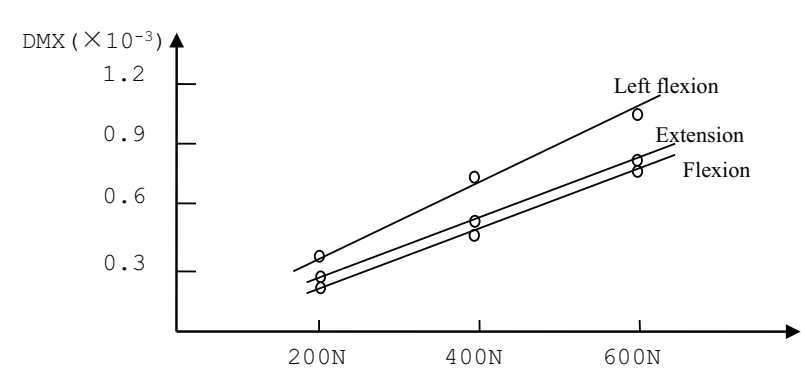

Fig. 12. The connection of maximum displacement and applied loadings.

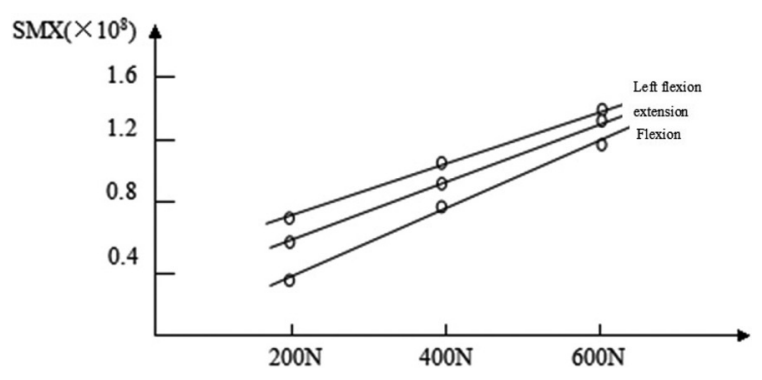

Fig. 13. The connection of maximum stress and applied loadings.

By changing the size of the applied loadings, and keeping the axial pressure and boundary conditions unchanged, we can get the connection of displacement, stress and applied loadings under three different stressed states, or the flexion movement, the extension movement and the left flexion movement, respectively. The effect of the right flexion movement is similar to that of the left flexion movement, and these connections are as shown in Figs 12 and 13.

The horizontal axes in Figs 12 and 13 show the size of the loading's values. The degree of deformation, the stress magnitude and the size of applied loadings of the vertebral show a linear relationship that is in accordance with a previously confirmed hypothesis that says that the material of the vertebral body is a linear, isotropic elastic material. From Figs 12 and 13, when the deformation of the vertebral body on the flexion movement is maximum, the stress on the vertebral body associated with extension movement is also maximum. With the addition of applied loadings, the stress of the vertebral body on flexion movement will gradually be increase towards a maximum, it is almost the same with clinical practice.

Cross-sections of the model yield qualitative strain contours plot of vertebral pulp when the model is under different states of motion (Figs 14-17).

These results indicate that the qualitative strain contours plot of nucleus pulpous of flexion movement focuses on the back, the strain of nucleus pulpous of extension movement focuses on the front, and that the strain of nucleus pulpous of flexion movement is focused on the opposite direction of movement. Stress is larger on the back of the nucleus pulpous when the vertebral body is in a state of flexion movement. Therefore, when annulus fibrosis is degenerating or under external loadings that are ictal, the 


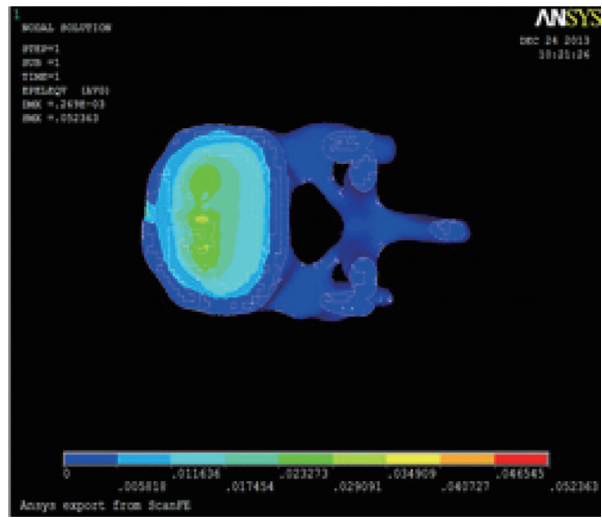

Fig. 14. The qualitative strain contours plot of vertebral pulp of flexion movement.

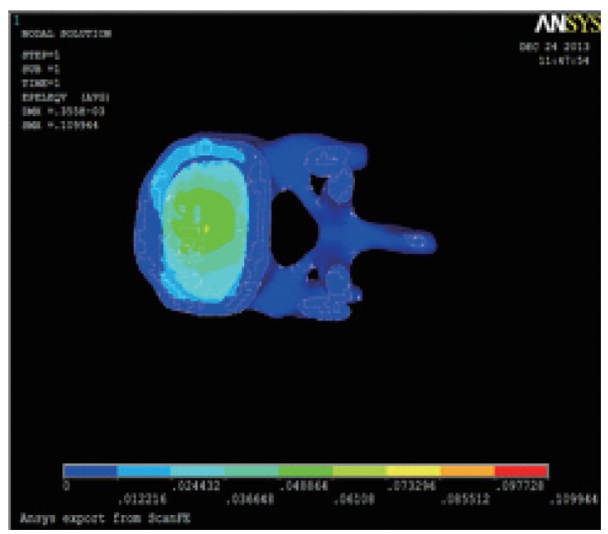

Fig. 16. The qualitative strain contours plot of vertebral pulp of left flexion movement.

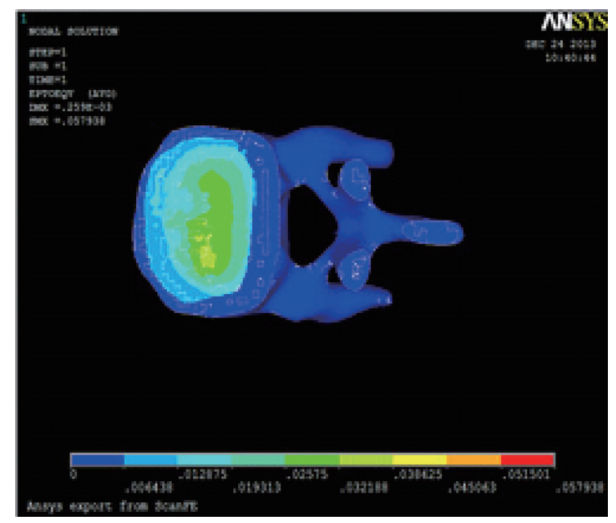

Fig. 15. The qualitative strain contours plot of vertebral pulp of extension movement.

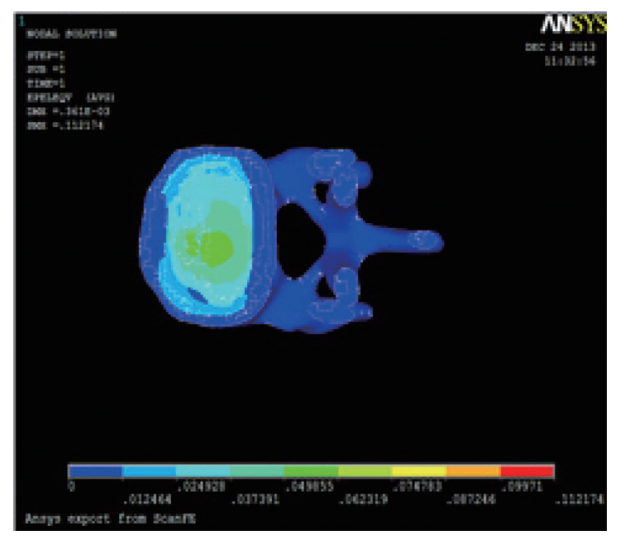

Fig. 17. The qualitative strain contours plot of vertebral pulp of right flexion movement.

annulus fibrosis will be in a state of protrusion and there will potential for other pathological changes, such as herniation of the nucleus pulpous.

\subsection{Finite element analysis of the degenerated model of lumbar vertebra L4-L5 sections}

In order to verify the validity of the finite element analysis results, simulation of the stress conditions found in degenerated models of lumbar vertebrae L4-L5 sections and investigations into the degenerated model through comparison with the normal model of the L4-L5 sections are used. Additionally, the stress conditions of the vertebral body and the small joints that are under four different states of motion are focused on.

\subsubsection{The construction of degenerated model of lumbar vertebrae L4-L5 sections}

Under normal conditions, the intervertebral disc is viscoelastic. However when degenerated, the viscoelasticity declines, such that spinal loadings are not normally distributed. Under dynamic loadings, there are several problems surrounding the organization of the cartilage plate, such as tiny fractures or weakened infiltration capacity. These problems can accelerate the degradation of the fiber ring and result 


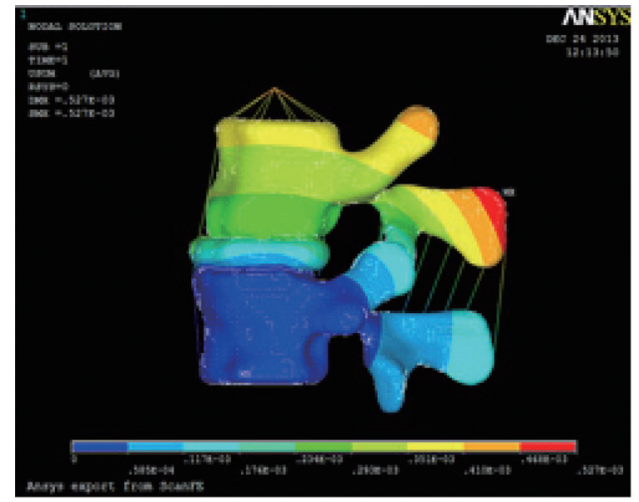

(a)

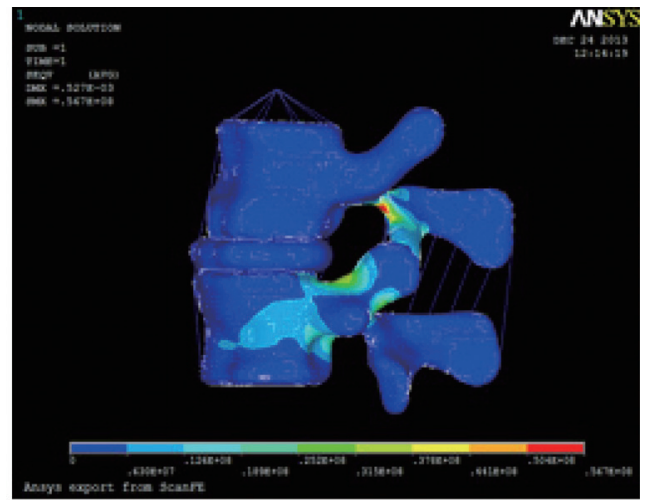

(b)

Fig. 18. (a) The qualitative displacement contours plot (b) The qualitative stress contours plot. The simulated results of flexion movement.

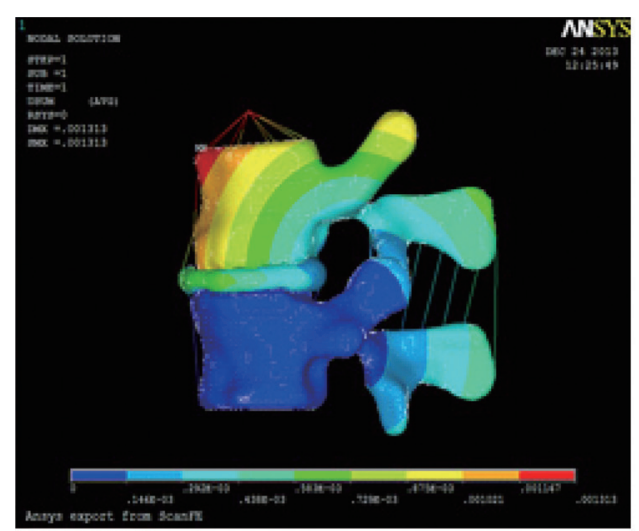

(a)

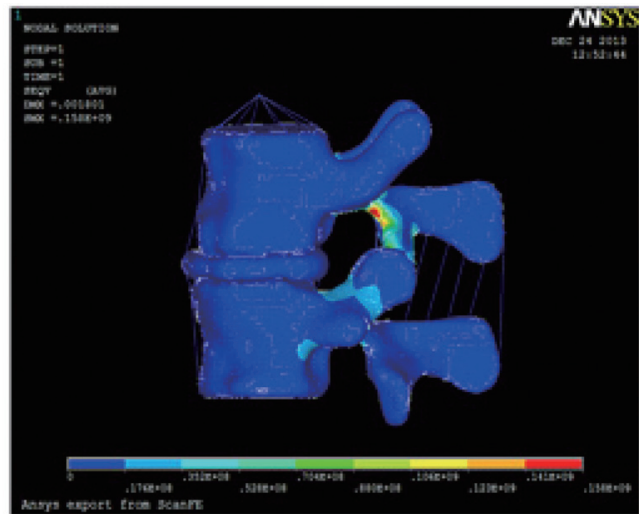

(b)

Fig. 19. (a) The qualitative displacement contours plot (b) The qualitative stress contours plot. The simulated results of extension movement.

in the protrusion of nucleus pulpous and can lead to the oppression of nerve roots, which can produce pain [9]. In later stages of degeneration, the change of geometrical morphology of the intervertebral disc is relatively larger. However, in early in the degenerative stage, the changes are only in the material properties. Here, only the early periods of degeneration of intervertebral disc, on the basis of normal model of L4-L5 sections, are studied by keeping the geometrical morphology unchanged and by modifying the material properties of the degenerated parts.

The degenerated sections are primarily the fiber ring and nucleus pulpous. Material properties that change following the fiber ring degeneration are [10]:

Young's modulus equals 3.0 MPa, Poisson ratio equals 0.45 .

The form of the normal model and that of Three-Dimensional FEA model which is degenerated are the same, they are as shown in Fig. 6.

\subsubsection{Stress analysis of the degenerated model of lumbar vertebra L4-15 sections}

Under the degenerated model, the same loading and boundary conditions as the normal model are used and simulate the following five movement states of the lumbar vertebrae: (1) flexion movement, 


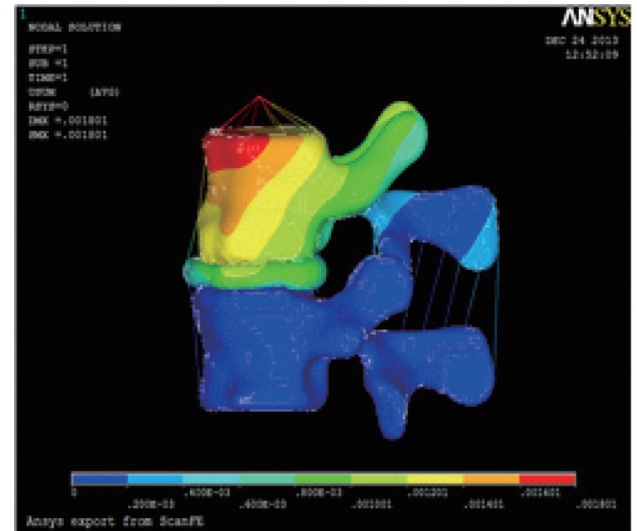

(a)

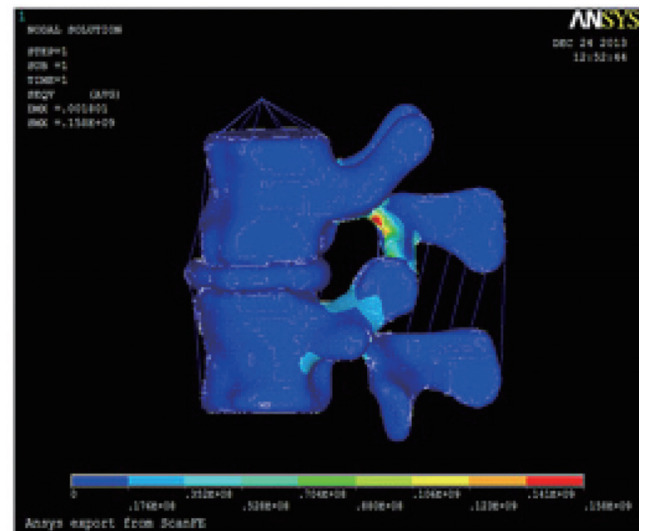

(b)

Fig. 20. (a) The qualitative displacement contours plot (b) The qualitative stress contours plot. The simulated results of left flexion movement.

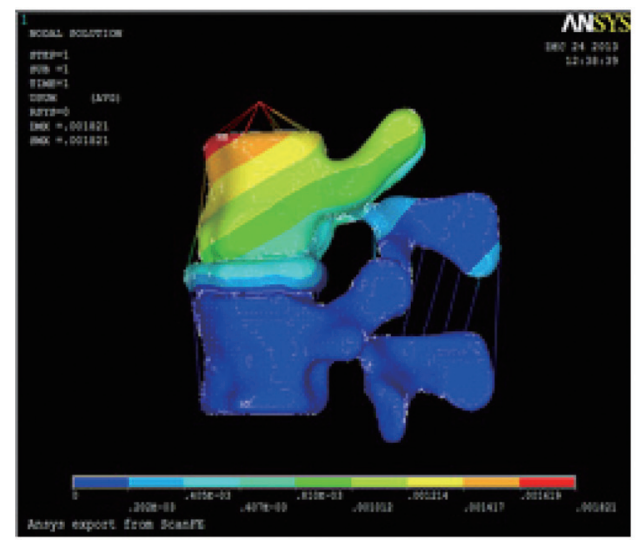

(a)

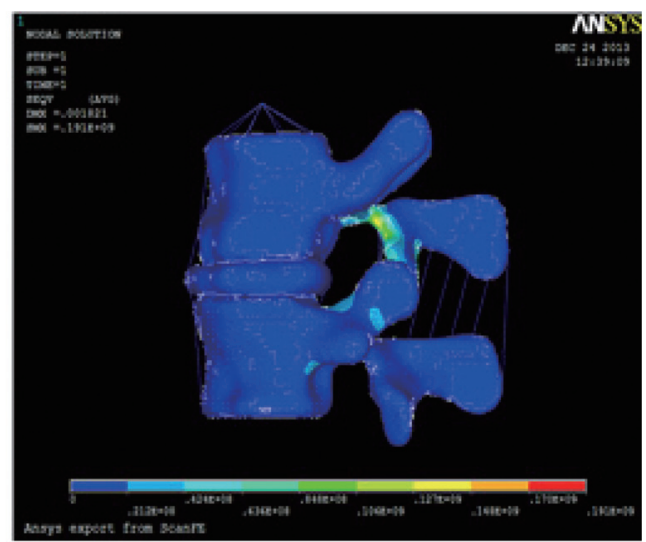

(b)

Fig. 21. (a) The qualitative displacement contours plot (b) The qualitative stress contours plot. The simulated results of right flexion movement.

(2) extension movement, (3) left flexion movement, (4) right flexion movement, and (5) axial rotational movement. The qualitative displacement and stress contours plots are shown in Figs 18-22.

\subsubsection{Analysis and comparison of the degenerated and normal models}

\subsubsection{The FE mechanics analysis of small joint under different states of motion}

The small joint is an important structure when the lumbar vertebrae is under stress. It is also helpful for the spine in resisting against the stressed states associated with flexion movement, extension movement and axial rotational movement. The intervertebral disc is an important part of the human body's bearing system has a close connection with the mechanical structure and function of small joints. In clinical studies, the degeneration of the intervertebral disc has lead to the damage of mechanical properties of small joints [11].

Under different states of movement, the stress status of the degenerated and normal model of small joint are shown in Figs 23-26. 


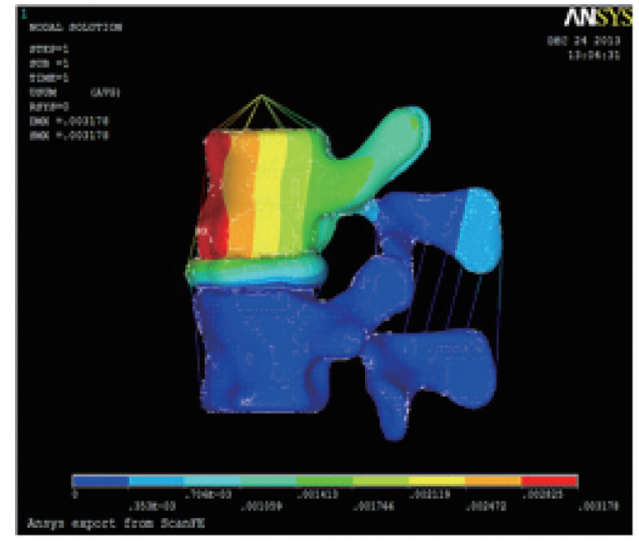

(a)

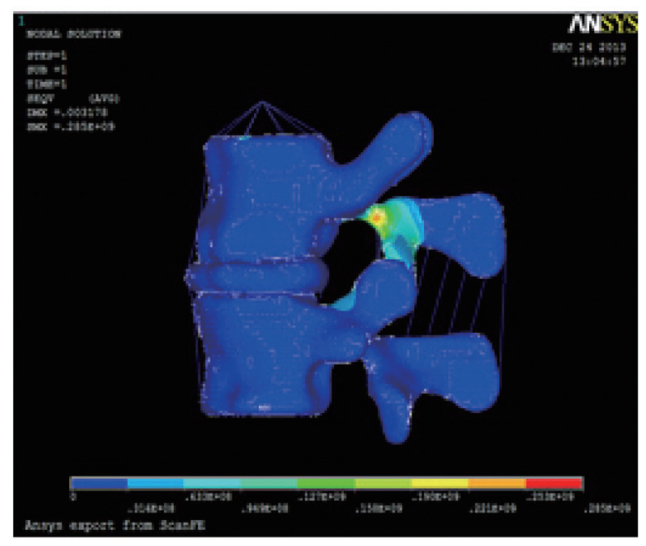

(b)

Fig. 22. (a) The qualitative displacement contours plot (b) The qualitative stress contours plot. The simulated results of axial rotational movement.

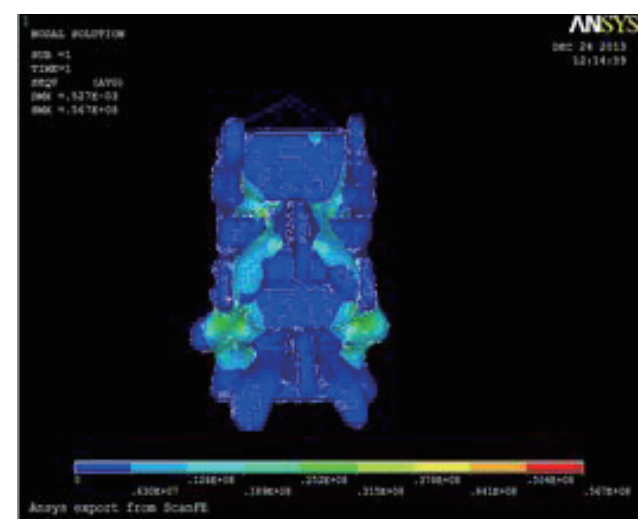

(a)

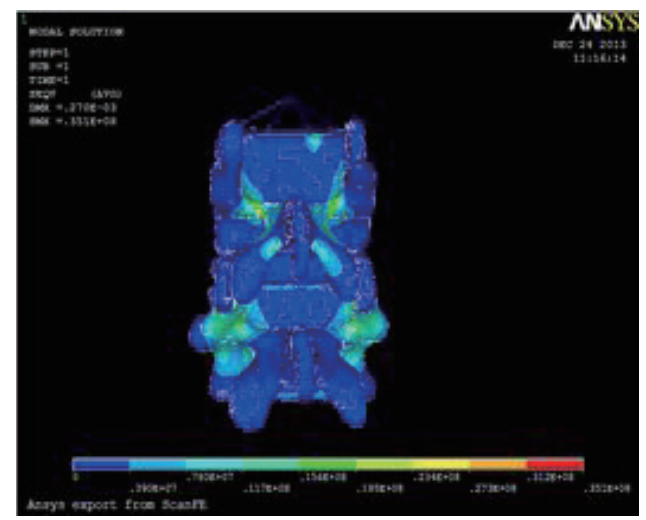

(b)

Fig. 23. The qualitative stress contours plot of flexion movement. (a) The normal model; (b) The degenerated model.

Observation has shown that the basic state of large stress in the small joints are caused by axial rotational movement and flexion movement, and states that have smaller stress are a result of flexion and extension movement, in all states of the normal model. When the spine is flexed or rotated, the stress exerted on the small joint is enlarged, supporting results found in clinical studies. Comparison of the stress associated with flexion and extension movement, the stress of the state of flexion movement is found to be smaller. When the vertebral body is in a flexion movement, most ligaments are extended. Spatially, the vertebral body approximately consider place near the small joints as the strong point. When rotation goes up along the vertical axis, it may only be extended by the anterior longitudinal ligament, on the state of extension motion, the stress of small joint that is considered as the strong point is also bigger.

With small joint stress under the normal model as a reference, when not in states of flexion or extension movements, small joint stress under the degenerated model is nearly identical to that seen under the normal model and it has a certain amount of growth. Following the degeneration of the lumbar vertebrae, the bearings of the small joints show large increases, especially under the state of flexion movement. Further, the resistance of the vertebral body against rotation movement decreases and the burden of 


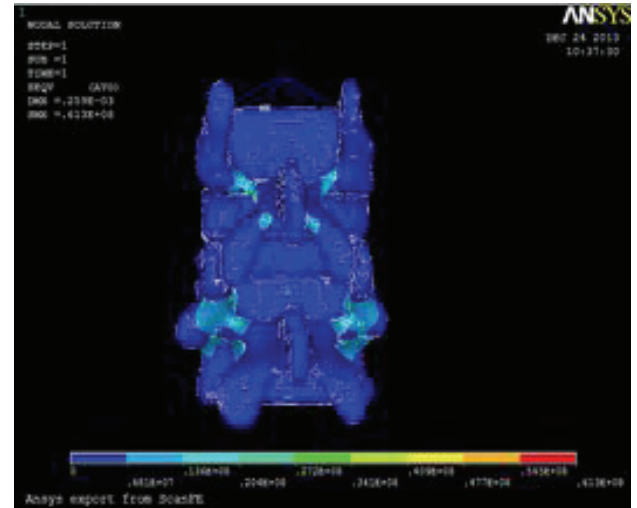

(a)

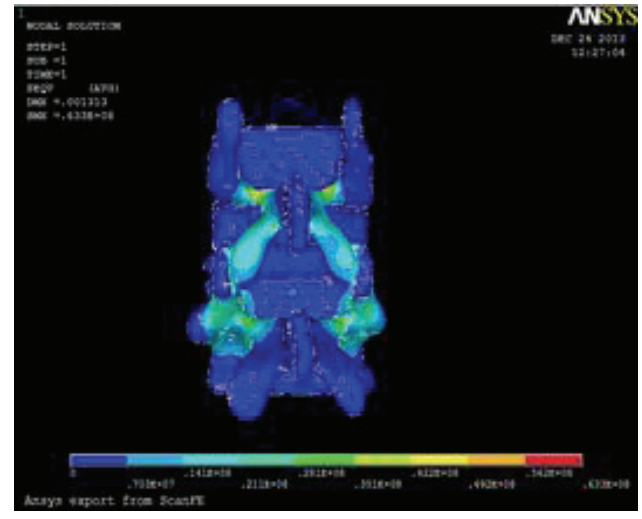

(b)

Fig. 24. The qualitative stress contours plot of extension movement. (a) The normal model; (b) The degenerated model.

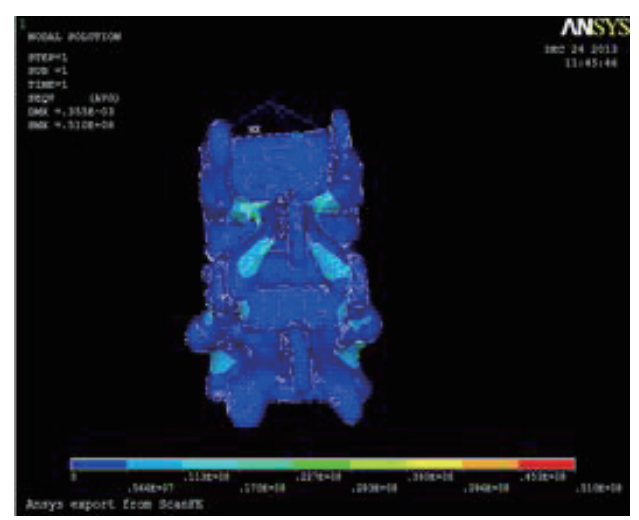

(a)

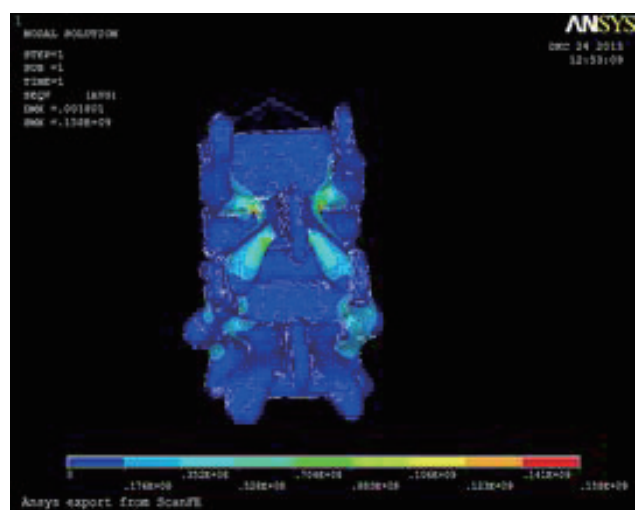

(b)

Fig. 25. The qualitative stress contours plot of left flexion movement. (a) The normal model; (b) The degenerated model.

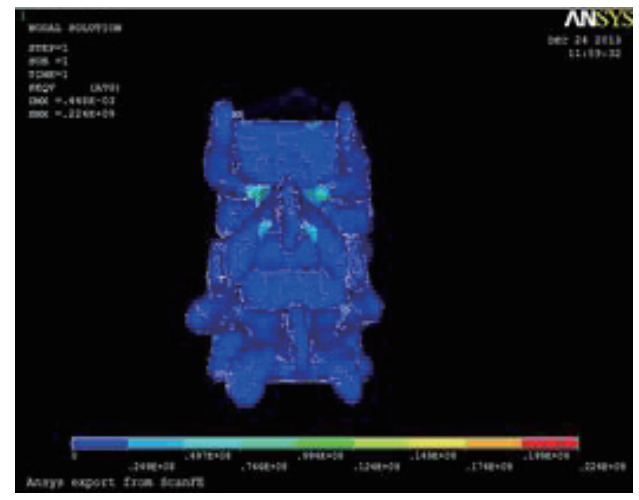

(a)

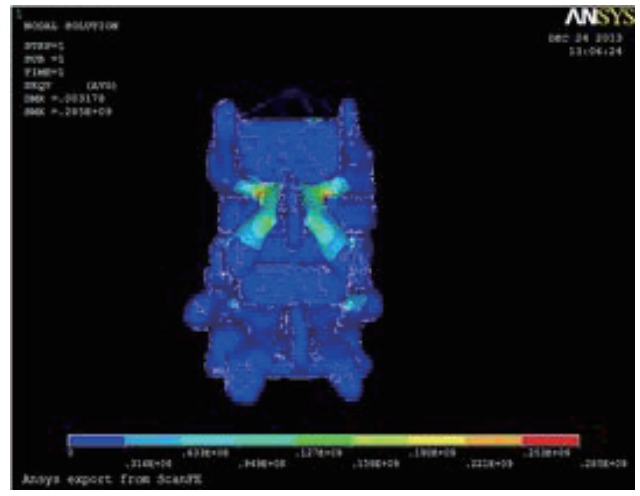

(b)

Fig. 26. The qualitative stress contours plot of axial rotational movement. (a) The normal model; (b) The degenerated model. 
Table 2

The stress value of normal model and that of degenerated model

\begin{tabular}{lcr}
\hline \multirow{2}{*}{$\begin{array}{l}\text { mates of } \\
\text { motion }\end{array}$} & $\begin{array}{c}\text { Normal model of } \\
\text { L4-L5 sections }\end{array}$ & $\begin{array}{r}\text { Degenerated model } \\
\text { of L4-L5 sections }\end{array}$ \\
\hline Flexion & $0.351 \mathrm{E}+08$ & $0.567 \mathrm{E}+08$ \\
Extension & $0.613 \mathrm{E}+08$ & $0.633 \mathrm{E}+08$ \\
Left flexion & $0.510 \mathrm{E}+08$ & $1.58 \mathrm{E}+08$ \\
Right flexion & $0.496 \mathrm{E}+08$ & $1.91 \mathrm{E}+08$ \\
Rotating & $0.224 \mathrm{E}+09$ & $0.285 \mathrm{E}+09$ \\
\hline
\end{tabular}

Table 3

The displacement values of normal model and degenerated model

\begin{tabular}{lcc}
\hline \multirow{2}{*}{$\begin{array}{l}\text { motes of } \\
\text { motion }\end{array}$} & \multicolumn{2}{c}{ DMX } \\
\cline { 2 - 3 } & $\begin{array}{c}\text { Normal model of } \\
\text { L4-L5 sections }\end{array}$ & $\begin{array}{c}\text { Degenerated model } \\
\text { of L4-L5 sections }\end{array}$ \\
\hline Flexion & $0.270 \mathrm{E}-03$ & $0.527 \mathrm{E}-03$ \\
Extension & $0.259 \mathrm{E}-03$ & $1.313 \mathrm{E}-03$ \\
Left flexion & $0.355 \mathrm{E}-03$ & $1.801 \mathrm{E}-03$ \\
Right flexion & $0.361 \mathrm{E}-03$ & $1.821 \mathrm{E}-03$ \\
Rotating & $0.448 \mathrm{E}-03$ & $3.178 \mathrm{E}-03$ \\
\hline
\end{tabular}

small joints increases greatly. Together, these effects can cause damage and calcification in small joints in the clinical practices.

\subsubsection{Finite element mechanics analysis of vertebral body under different states of motion}

Qualitative displacement observations and stress contours plots of the normal and degenerated models that are under different states of motion give the results found in Tables 2 and 3.

Whether it is the normal or degenerated model, the stress value is largest when the front is flexing forward, the back is extending and the right is in a state of right flexion motion, matching what has been shown in clinical practices. For the degenerated model, the distribution of the stress value is similar to that of the normal model, and the specific value of stress is relatively bigger. However, the gap of displacement values between these two models is significant. For rotational and extension movements, the changing scope of stress is the smallest and the stress influence of the intervertebral disc is the smallest. Therefore, we can conclude that rotational and extension movements are not the main causes of intervertebral disc diseases.

After the comprehensive analysis, the results indicate that stress and displacement values of degenerated model of L4-L5 sections are bigger than that of normal model after the intervertebral disc degenerated. The change will more easily lead to the retroposition of nucleus pulpous and deformation of the intervertebral disc, and then result in a series of pathological changes by oppressing nerves, such as the waist pain, the hyperplasia of vertebral bone, the herniation of lumbar intervertebral disc.

\section{Conclusions and expectations}

Based on the understanding of structure features of spinal lumbar vertebrae and characteristics of CT images, some discussion has been made on the method of building Three-Dimensional model and finite element mesh model of spinal lumbar vertebrae. This discussion has led to situations which test stress, strain and deformation when the normal model and degenerated model are under different states of motion by using the FEA software ANSYS. When simulated and analyzed, the validity of model data is compared and demonstrated. The results have certain reference values to diagnosis and treatment of spinal lumbar vertebrae diseases that are in the high incidence, and are also helpful for researchers that are connected with surgical sciences.

\section{Acknowledgement}

This research is supported by the NSFC Grant No. 61172147. The Fundamental Research Funds for the Central Universities Grant No. K5051303005, K5051303015. 


\section{References}

[1] Zeng P., The basic tutorial of Finite element analysis, Publishing House of Tsinghua University, 2008.

[2] Lorensen W.E., Harvey E.C., Marching C., A High Resolution 3D Surface Construction Algorithm[J], ACM Siggraph Computer Graphics, 1987, 21(04), 163-169.

[3] Qian H.Y., Three-Dimensional medical image segmentation method research, Southeast University, 2007.

[4] Song X., Cheng M., Wang B.L., etc, Automatical liver segmentation method with incredible link[J], Journal of Graphics and Computer-Aided Design, 2012, 9, 24(9), 1188-1192.

[5] Simpleware. Simpleware Tutorial Guide, 2010.

[6] Zeng X.L., Peng L., Bai J., Three-Dimensional finite element modeling and analysis of spinal lumbar vertebrae L4-L5 sections based on CT data[J], Biomedical Engineering, Beijing, 2007, (32), 266-269.

[7] Wang Z., Wang Z.Y., Wang D.M., etc, The construction of the overall FEA model based on he thoracic lumbar segment and sacrum CT images[J], Publishing house of machinery, 2007, 4(23), 24-26.

[8] Renner S.M., Natarajan R.N., Patwardhan A.G. et al., Novel model to analyze the effect of a large compressive follower preload on range of motions in a lumbar spine[J], Journal of Biomechanics, 2007, 22(9), 988-998.

[9] Wang Z.Y., Application and progress of finite element method in Spinal biomechanics. Journal of Shanghai Jiaotong University, 2007, 27(9), 1156-1159.

[10] Guo L.X., Chen W., Liu X.Y., Dynamic characteristics analysis of human injured spine based on finite element model[J], Journal of Northeastern University, 2005, 26(9), 836-839.

[11] Daniel K., Jan W., Robert S., Wolfram Mittelmeier and Bader. The application of finite element method in the orthopaedic biomechanics[D], University of Rostock, Department of Orthopacdics Rostock, Germany, 2010. 